\title{
A Cross-sectional Study of Alcohol Drinking and Health-related Quality of Life among Male Workers in Japan
}

\author{
Isao Saito ${ }^{1}$, Tomonori Okamura ${ }^{2}$, Shunichi Fukuhara ${ }^{3}$, Taichiro Tanaka ${ }^{2}$, \\ Yoshimi Suzukamo ${ }^{3}$, Akira Okayama ${ }^{4}$, Hirotsugu Ueshima ${ }^{2}$ and the HIPOP-OHP Research \\ Group 5
}

${ }^{1}$ Department of Public Health, Nara Medical University, ${ }^{2}$ Department of Health Science, Shiga University of Medical Science, ${ }^{3}$ Department of Epidemiology and Healthcare Research, Graduate School of Medicine and Public Health, Kyoto University, ${ }^{4}$ Department of Preventive Cardiology, National Cardiovascular Center and ${ }^{5}$ Investigators of the research group are listed in the Appendix, Japan

\begin{abstract}
A Cross-sectional Study of Alcohol Drinking and Health-related Quality of Life among Male Workers in Japan: Isao SAITo, et al. Department of Public Health, Nara Medical UniversityBackground: Although light and moderate alcohol drinkers are likely to have better subjective health, the sub-scales for subjective health have not been well documented. Methods: We studied 4,521 male workers aged $25 \mathrm{yr}$ and older with no history of cancer or cardiovascular disease, in 12 occupational groups in Japan. Data were from the High-risk and Population Strategy for Occupational Health Promotion Study (HIPOP-OHP). Drinking status was classified according to daily alcohol intake or frequency of drinking. We assessed the health-related quality of life (HRQOL) based on scores for five scales of the SF-36. Results: Decreased odds ratios of sub-optimal HRQOL conditions, defined as less than the median SF-36 scores, for Role-Physical and General Health were found among persons who consumed 1.0 to 22.9 $\mathrm{g} / \mathrm{d}$ of alcohol. Odds ratios for sub-optimal Vitality conditions were lowered according to increased levels of alcohol intake. Role-Emotional scores were not associated with alcohol drinking. People who drank 5 to $6 \mathrm{~d} / \mathrm{wk}$ had higher levels of Role-Physical and Vitality, and those who drank 1 to $2 \mathrm{~d} / \mathrm{wk}$ had better Vitality and Mental Health scores than non-drinkers. When adjusted for age, marital status, working hours, physical activity at work, self-reported job stress, smoking, regular exercise, hypertension, hyperlipidemia, and diabetes, the associations were almost unchanged except for General Health. Conclusions: Associations
\end{abstract}

Received Jun 6, 2005; Accepted Sep 6, 2005

Correspondence to: I. Saito, Department of Public Health, Nara Medical University, 840 Shijo-cho, Kashihara, Nara 634-8521, Japan (e-mail: saitoi@naramed-u.ac.jp) of drinking patterns with subjective health varied in five sub-scales of the SF-36. Overall, alcohol drinkers rated their health as good in comparison with non-drinkers. (J Occup Health 2005; 47: 496-503)

Key words: Alcohol drinking, Epidemiology, Healthrelated quality of life, SF-36, Subjective health

It is widely known that light and moderate alcohol intake are associated with decreased risk of incident cardiovascular disease $^{1-3)}$ and all-cause mortality ${ }^{4-6)}$. The mechanism for this protective effect has been postulated to be due to the modification of high-density lipoprotein ${ }^{7)}$ and platelet aggregability ${ }^{8,9)}$ and lowered fibrinogen levels ${ }^{10)}$.

Several epidemiological studies have indicated that people with higher levels of self-rated health ${ }^{11-14)}$ or good health practices ${ }^{15,16)}$ are at low risk of mortality and cardiovascular disease. Moreover, a few studies have suggested that light and moderate drinkers rate their health as $\operatorname{good}^{17-20)}$. Among Japanese employees, it was documented that men who consumed 25 to 35 or $49 \mathrm{~g} / \mathrm{d}$ or more of alcohol had a significantly lower risk of selfrated ill health compared with non-drinkers ${ }^{20)}$. This Japanese study equated ill health with the response of "poor" on the self-reported questionnaire and did not separate abstainers in the analysis, but only $4.8 \%$ of the study participants were in this category. Therefore, the inverse association might be due to a selection bias for healthy people who consumed a lot of alcohol. Furthermore, previous studies on this issue did not consider sub-scales for subjective health. So, it is important to better understand the effect of alcohol on subjective physical and mental health as measured in a health-related quality of life (HRQOL) assessment. 
The Medical Outcomes Study Short-Form 36-Item Health Survey (SF-36) is one of the generic HRQOL instruments. SF-36 is based on a conceptual model consisting of physical and mental health constructs, and it is designed to measure perceived health status and daily functioning. It consists of 36 items that are scored in the following eight domains: Physical Functioning, RolePhysical, Bodily Pain, General Health, Vitality, Social Functioning, Role-Emotional, and Mental Health. The score on each scale ranges from 0 to 100 , with a low score indicating poor health or great disability. SF-36 is widely used and is available in many languages, including Japanese ${ }^{21,22)}$.

In the present study, we examined the association between alcohol drinking patterns and the HRQOL among male workers, controlling for working environment, health practices, and burden of common chronic diseases.

\section{Methods}

\section{Population}

We analyzed baseline data from the High-risk and Population Strategy for Occupational Health Promotion (HIPOP-OHP) intervention study ${ }^{23)}$. This study population consisted of 12 companies (one life insurance company, two chemical companies, one electrical appliance company research laboratory, and eight electrical appliance manufacturers). There were 5,002 male workers, aged $25 \mathrm{yr}$ and over, who responded to an assessment of drinking habits at the baseline examination during 1999-2000. The response rate was 92\% (5,002/ 5,442 ), and there were no differences among companies. We selected 4,521 male workers, after exclusion of those who did not reply to an answer on alcohol consumption $(n=307)$, those who had a history of cancer or cardiovascular disease $(n=96)$, and those who did not complete the SF-36 questionnaire $(n=106)$. In all, 481 men were excluded from our analysis (28 overlapped) due to our exclusion criteria. Their mean age (42.4 y) was slightly higher than the mean age of the other. Adjusted for age, a higher proportion of current smokers $(57.6 \%)$ and a lower proportion of those who did regular exercise $(58.9 \%)$ were listed among them. However, there were no differences in proportions of married persons, daytime workers, persons with heavy physical activity at work, persons perceiving job stress, and people with obesity, hypertension, hyperlipidemia, and/or diabetes, between excluded and analyzed subjects.

Written informed consent was obtained from the subjects for individual intervention. Since the HIPOPOHP study was designed as an occupational communitybased intervention by means of population strategy, we did not think it necessary to obtain written informed consent from all individuals. However, we informed them that the data were being used in our study and individual information was strictly protected under the privacy policy. Furthermore, approval for this study was obtained from the Institutional Review Board of Shiga University of Medical Science (No. 10-16).

\section{Measurements}

In the HIPOP-OHP study, we used the same questionnaire to get health information from all companies in accordance with the common protocol described elsewhere ${ }^{23)}$. At each company, after a 5-min rest timed with an hourglass, subjects' blood pressure was measured twice using an automatic sphygmomanometer. The mean of these two values was used. Hypertension was defined as systolic blood pressure (SBP) of 140 $\mathrm{mmHg}$ or higher or diastolic blood pressure (DBP) of 90 $\mathrm{mmHg}$ or higher. Serum gamma-glutamyl-transpeptidase activity $(\gamma$-GTP) was measured using a colorimetric method. Lipid measurements, including HDL (highdensity lipoprotein) cholesterol, were standardized according to the protocol of the U.S. Cholesterol Reference Method Laboratory Network (CRMLN) of the Centers for Disease Control and Prevention to compare values among laboratories participating in the study. Subjects with hyperlipidemia and diabetes were defined as those who had ever been diagnosed by a physician, regardless of treatment status. Body mass index (BMI) was calculated using measured weight $(\mathrm{kg})$ divided by the square of the height $\left(\mathrm{m}^{2}\right)$.

Each subject's drinking habits were assessed using a previously published method ${ }^{24)}$. First, we asked them the following: "Could you choose the appropriate description of your alcohol consumption in the previous month: (1) never drank, (2) drank in the past, or (3) current drinker?" In the case of current drinkers, we asked them, "How many times per week do you usually drink alcohol? Which alcoholic beverages do you drink on a typical occasion?" and "Please describe the typical quantity of each beverage." The frequency of alcohol consumption during a week and the total alcohol intake on each occasion were determined and used to calculate the alcohol intake per week. We defined the ethanol concentration of each major alcoholic beverage as follows: beer 5\%, sake 16\%, whiskey $40 \%$, shochu $25 \%$, and wine $12 \%$. Happo-shu, which has a taste and ethanol concentration similar to that of beer but includes less malt as a raw material, was calculated as beer. The ethanol concentration of other minor beverages was defined individually. This value was then divided by 7 to obtain the average alcohol intake per day. Drinkers were defined as those consuming more than 0.3 gou a week $(1.0 \mathrm{~g} / \mathrm{d}$ of ethanol), as in previous cohort studies in Japan ${ }^{2,3)}$. So, self-described current drinkers were re-classified as nondrinkers if they reported consuming less than 0.3 gou a week. The reason why we used frequency of alcohol drinking as a variable was that it was considered to be an important marker indicating drinking behavior, and the 
frequency itself has been significantly associated with cardiovascular disease events ${ }^{1}$. We classified the responses into six groups, i.e., non-drinker, ex-drinker, and four categories of current drinkers according to alcohol intake per day: 1.0 to $22.9 \mathrm{~g}, 23.0$ to $45.9 \mathrm{~g}, 46.0$ to $68.9 \mathrm{~g}$, and $69.0 \mathrm{~g}$ and over. These groups correspond to the categories related to incidents of coronary heart disease and stroke among Japanese ${ }^{3)}$.

We assessed individuals' marital status, working hours, physical activity at work, self-reported job stress, smoking status, and regular exercise and added these variables to the multivariate models as confounders.

The assessment of HRQOL was done with version 2.0 of the SF-36 questionnaire form and scoring program ${ }^{25)}$. The Japanese version of the SF-36 has been validated in previous studies ${ }^{26)}$. Missing data were complemented in the validated algorithm of calculation. In addition, we calculated scores by the norm based scoring (NBS) method, which was set at 50 for Japanese means based on the normal distribution of the scores derived from the SF-36 national survey in 2002. The first step in the NBS consists of standardizing each SF-36v2 scale using a $\mathrm{Z}$ score transformation. A $\mathrm{z}$-score for each scale is computed by subtracting the 2002 general Japanese population mean for each SF-36 scale and dividing the difference by the corresponding scale standard deviation from the 2002 general Japanese population. The second step involves transforming each SF-36v2 z-score to the NBS $(50,10)$. This is accomplished by multiplying each z-score from Step 1 by 10 and adding the resulting product to 50 .

Although the SF-36 has eight sub-scales, we used only the five sub-scales of Role-Physical, General Health, Role-Emotional, Mental Health, and Vitality, because the HIPOP-OHP study basically was conducted for healthy workers without physical disability and they were mostly middle-aged or younger men; the other three sub-scales (Bodily Pain, Social Functioning, and Physical Functioning) were not investigated ${ }^{23)}$.

Higher levels of Role-Physical and Role-Emotional represent the conditions where people can work usually without physical and psychological problems, respectively. General Health is assessed by self-perceived health status; for example, "I am as healthy as anybody I know." Higher scores of Vitality indicate conditions in which people have a lot of energy or are not exhausted at all. Mental Health reflects feelings of depression, nervousness, and happiness. Each sub-scale score consisted of three to five questions in the SF-36.

Overall, the SF-36 sub-scales were divided into two domains representing physical and mental health. In a validation study of the Japanese version, Role-Physical was interpreted as a condition of physical health, and Mental Health and Vitality were valid scales representing mental health. General Health and Role-Emotional scales were not consistent with hypotheses, but the validation study suggested that General Health reflected both physical and mental conditions, and Role-Emotional was closely related to the physical component ${ }^{27)}$. We defined sub-optimal HRQOL as less than the median score of all subjects for each SF-36 sub-scale.

To test the internal consistency reliability, we computed Cronbach's alpha for each SF-36 sub-scale. These ranged between 0.75 and 0.91 . All coefficients were satisfied with criteria $(>0.7)$ that were considered to be reliable for the use of group level comparison.

\section{Data Analysis}

Means of alcohol consumption and the frequency of drinking were computed by the levels of alcohol consumption. Also, we calculated the proportions of married persons, daytime workers, persons with heavy physical activity at work, persons perceiving job stress, current smokers, those who did regular exercise, and people with obesity, hypertension, hyperlipidemia, and/ or diabetes. Means of scores of the original SF-36 and the NBS by sub-scales and standard deviations were computed by drinking status.

Age-adjusted and multivariate logistic models were done. The risk of sub-optimal HRQOL based on SF-36 was calculated according to alcohol consumption and frequency of alcohol drinking in comparison with nondrinkers. The odds ratios were adjusted for age in model 1. In model 2, marital status (married, other), working hours (daytime, other), physical activity at work (heavy, other), self-reported job stress (yes, no), smoking status (current smoker, other), and regular exercise (yes, no) were added using dummy variables. Finally, we added factors indicating obesity, hypertension, hyperlipidemia, and diabetes to model 2. This became model 3. All analyses were done using SAS software, version 8.2 (SAS Institute, Inc., Cary, North Carolina).

\section{Results}

Among 4,521 male workers (mean age, 39.4 y), 60.4\% were current drinkers. Table 1 shows the characteristics of our subjects by group. Frequency of drinking increased as the amount of alcohol consumption increased. Compared to non-drinkers and ex-drinkers, subjects who consumed the lowest amount of alcohol had better health practices, i.e., lower prevalence of smoking and higher proportion of regular exercise. High percentages of obesity and diabetes were seen among ex-drinkers. Means of HDL-cholesterol were clearly elevated in accordance with alcohol consumption levels. People who reported consumption of 1.0 to $22.9 \mathrm{~g} / \mathrm{d}$ of alcohol had higher scores in the areas of Role-Physical, whereas exdrinkers tended to have low scores, especially in General Health. Vitality scores were higher among men who drank more. The NBS scores by sub-scales are presented. 
Table 1. Population characteristics by alcohol consumption among male workers in the HIPOP-OHP Study

\begin{tabular}{|c|c|c|c|c|c|c|}
\hline \multirow[b]{2}{*}{ Variables } & \multirow[t]{2}{*}{ Non-drinker } & \multirow[t]{2}{*}{ Ex-drinker } & \multicolumn{4}{|c|}{ Current drinkers by alcohol consumption, g/d } \\
\hline & & & $1.0-22.9$ & $23.0-45.9$ & $46.0-68.9$ & 69.0 and over \\
\hline Number, $\mathrm{n}$ & 1,497 & 291 & 1,408 & 703 & 359 & 263 \\
\hline Mean age, y & 37.8 & 39.0 & 38.6 & 41.3 & 43.4 & 42.5 \\
\hline Mean $\left(\mathrm{SD}^{\S}\right)$ alcohol consumption, $\mathrm{g} / \mathrm{d}$ & - & - & $12.1(5.8)$ & $33.2(6.5)$ & $54.8(6.2)$ & $93.6(26.0)$ \\
\hline Frequency of alcohol drinking, $\mathrm{d} / \mathrm{wk}$ & - & - & 4.0 & 5.8 & 6.4 & 6.6 \\
\hline Married, \% & 67.1 & 72.0 & 76.7 & 79.6 & 83.4 & 83.0 \\
\hline Working hours, $\%$ daytime & 60.3 & 61.9 & 73.3 & 69.5 & 71.3 & 61.1 \\
\hline Physical activity at work, $\%$ heavy & 6.9 & 9.4 & 6.3 & 8.6 & 9.0 & 6.6 \\
\hline Self-reported job stress, $\%$ yes & 22.6 & 23.0 & 22.5 & 24.3 & 23.5 & 21.5 \\
\hline Current smokers, $\%$ & 49.3 & 56.8 & 46.3 & 59.8 & 60.7 & 71.4 \\
\hline Regular exercise, $\%$ & 58.9 & 60.2 & 64.7 & 64.5 & 66.1 & 56.9 \\
\hline Obesity*, \% & 23.6 & 30.9 & 20.5 & 19.8 & 24.2 & 26.6 \\
\hline Hypertension**, \% & 10.2 & 16.8 & 13.7 & 15.3 & 24.3 & 19.9 \\
\hline Hyperlipidemia $^{\dagger}, \%$ & 12.6 & 13.8 & 11.1 & 14.6 & 15.7 & 16.4 \\
\hline Diabetes $^{\dagger}, \%$ & 4.8 & 10.4 & 5.3 & 6.3 & 7.6 & 7.7 \\
\hline Mean HDL-cholesterol ${ }^{\ddagger}\left(\mathrm{SD}^{\S}\right), \mathrm{mg} / \mathrm{dL}$ & $52.2(12.6)$ & $51.9(12.5)$ & $55.7(13.6)$ & $59.0(13.9)$ & $59.0(13.4)$ & $60.3(14.9)$ \\
\hline \multicolumn{7}{|l|}{ Mean SF-36 scores $\left(\mathrm{SD}^{\S}\right)$ by scales } \\
\hline Role-Physical & $86.6(19.1)$ & $83.6(21.0)$ & $89.0(17.2)$ & $86.5(20.0)$ & $87.6(17.8)$ & $87.4(18.8)$ \\
\hline General Health & $58.2(16.9)$ & $54.9(15.2)$ & $60.3(16.3)$ & $59.4(16.2)$ & $57.9(15.9)$ & $60.6(16.6)$ \\
\hline Vitality & $52.2(18.9)$ & $52.3(17.5)$ & $53.9(18.2)$ & $54.5(17.8)$ & $55.7(18.3)$ & $56.4(18.9)$ \\
\hline Role-Emotional & $85.8(20.0)$ & $84.4(21.6)$ & $87.5(18.0)$ & $86.3(19.7)$ & $87.0(18.6)$ & $87.2(19.2)$ \\
\hline Mental Health & $65.4(17.3)$ & $64.7(17.1)$ & $66.8(16.8)$ & $66.0(17.3)$ & $65.9(17.6)$ & $67.0(17.1)$ \\
\hline \multicolumn{7}{|l|}{ Mean SF-36 NBS scores $\|\left(\mathrm{SD}^{\S}\right)$ by scales } \\
\hline Role-Physical & $48.9(10.4)$ & $47.3(11.5)$ & $50.2(9.4)$ & $48.9(10.9)$ & 49.5 (9.7) & $49.4(10.2)$ \\
\hline General Health & $46.9 \quad(9.1)$ & $45.1 \quad(8.2)$ & $48.0(8.8)$ & $47.5 \quad(8.7)$ & $46.7(8.6)$ & $48.2 \quad(8.9)$ \\
\hline Vitality & $45.2 \quad(9.3)$ & $45.2 \quad(8.6)$ & $46.0(8.9)$ & 46.3 (8.8) & $46.9(9.0)$ & $47.3 \quad(9.3)$ \\
\hline Role-Emotional & $49.3(10.2)$ & $48.6(11.0)$ & $50.2(9.2)$ & $49.6(10.0)$ & $50.0(9.5)$ & $50.0 \quad(9.8)$ \\
\hline Mental Health & $46.7 \quad(9.2)$ & $46.3 \quad(8.9)$ & $47.4(8.9)$ & $47.0 \quad(9.2)$ & $46.9(9.4)$ & $47.5 \quad(9.1)$ \\
\hline
\end{tabular}

*Defined as body mass index $\geq 25 \mathrm{~kg} / \mathrm{m}^{2}$. **Defined as systolic blood pressure $\geq 140 \mathrm{mmHg}$, diastolic blood pressure $\geq 90 \mathrm{mmHg}$, or current use of antihypertensive medication. Defined as those who had been told by doctors. *Data were available for 3,310 subjects. ${ }^{\S}$ Standard deviation. $\|$ NBS (Norm-based scoring) scores, which were set at 50 as Japanese means, were based on the total distribution from the SF-36 national survey ${ }^{25}$.

Compared with Japanese means, which were set at 50, our population of SF-36 means in each sub-scale were below 50 .

Age-adjusted and multivariate odds ratios, according to daily consumption of alcohol, for sub-optimal health are shown by SF-36 sub-scales in Table 2. The ageadjusted odds ratio for General Health was 1.69 (95\% confidence interval, 1.29-2.20) in ex-drinkers. The group that consumed 1.0 to $22.9 \mathrm{~g} / \mathrm{d}$ of alcohol had a low risk for sub-optimal scores in Role-Physical, General Health, and Vitality. Those for Vitality were lowered in accordance with increasing levels of alcohol intake. Among those who reported heavy drinking $(69.0 \mathrm{~g} / \mathrm{d}$ and over), the odds ratio did not increase at all. In models 2 and 3, after addition of confounding factors, alcohol drinkers who consumed 1.0 to $22.9 \mathrm{~g} / \mathrm{d}$ were more likely to have a good HRQOL; however, the odds ratio for
General Health was not statistically significant after the adjustment. Inverse association of alcohol consumption with Vitality scores still remained significant in models 2 and 3.

Table 3 shows age-adjusted and multivariate odds ratios for sub-optimal HRQOL by the frequency of alcohol drinking per week. Alcohol consumption levels by four drinking frequency categories corresponded with 9.9, $18.7,30.8$, and $45.8 \mathrm{~g} / \mathrm{d}$. Individuals who consumed alcohol 1 to $2 \mathrm{~d} /$ wk had higher HRQOL levels for General Health, Vitality, and Mental Health, and those who consumed alcohol on 5 to $6 \mathrm{~d} / \mathrm{wk}$ were in good condition as determined by Role-Physical. People who drank alcohol 3 to 4 or 5 to $6 \mathrm{~d} /$ wk had good HRQOL Vitality scores. When the odds ratios were adjusted for several factors in models 2 and 3, the association was almost the same. 

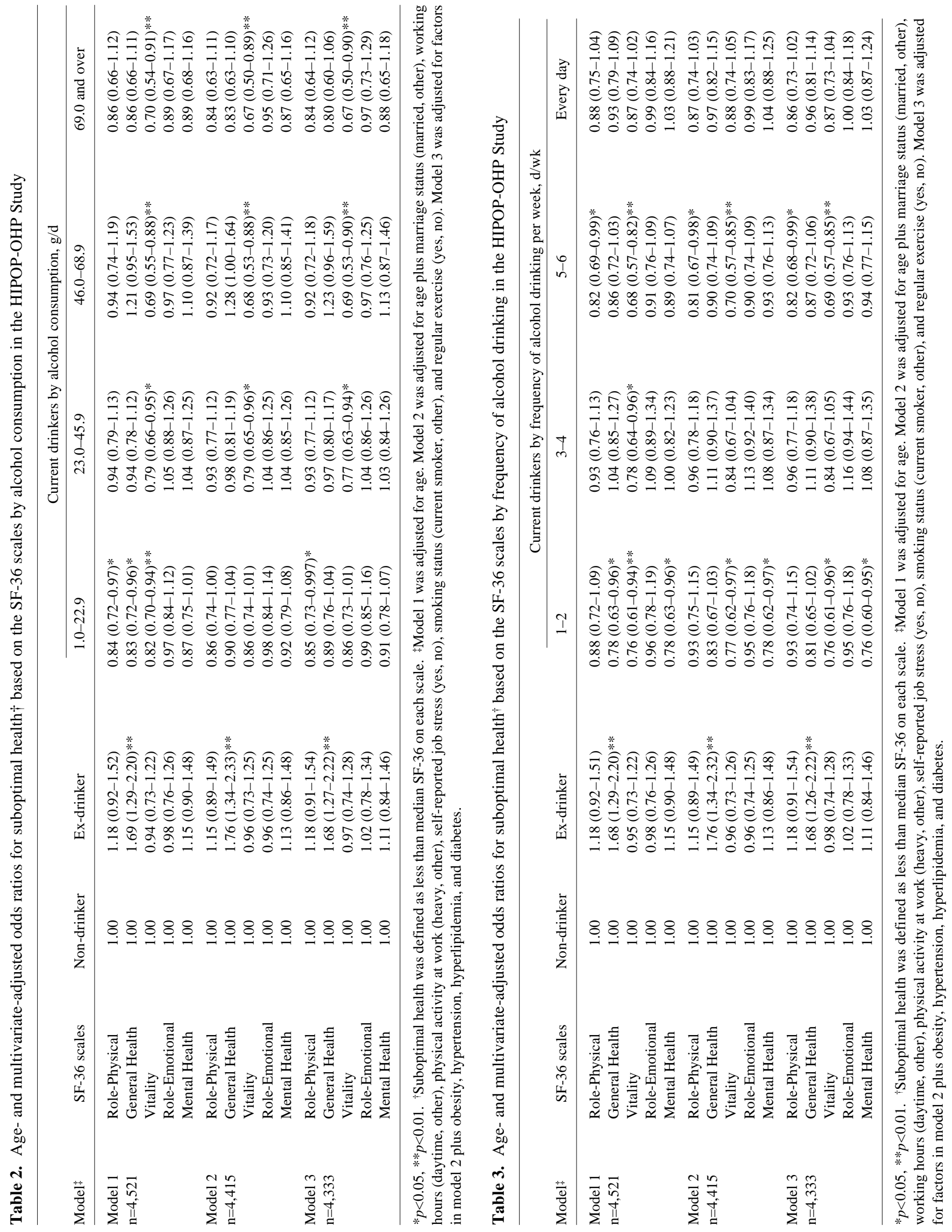
Since it was possible that our data were biased by heavy or frequent drinkers who had poor health due to alcoholic liver disease, we re-analyzed the data excluding subjects with levels of $\gamma$-GTP greater than 100 IU/L. Furthermore, considering effects of common chronic diseases, such as obesity, hypertension, hyperlipidemia, and diabetes, we examined the data among only healthier men without the diseases. Regardless of chronic disease conditions, we found similar associations to those shown in Tables 2 and 3 (data not shown).

\section{Discussion}

In the present study, we used the baseline dataset of an intervention trial (HIPOP-OHP study) ${ }^{23}$. The population strategy of the HIPOP-OHP study was conducted in three fields, i.e., nutrition, physical activity, and smoking from 1999 to 2004. Although intervention for alcohol intake was defined as one part of the population strategy for the nutrition field, there was no announcement regarding alcohol intervention, at least in the baseline survey in all companies. Accordingly, our results were not affected by the intervention process.

We found that people who consumed 1.0 to $22.9 \mathrm{~g} / \mathrm{d}$ of alcohol scored high in HRQOL conditions: RolePhysical, General Health, and Vitality. Also, vitality conditions were better in accordance with increased levels of alcohol intake. The risks for sub-optimal health did not increase even among heavy drinkers $(69.0 \mathrm{~g} / \mathrm{d}$ and over). Ex-drinkers were at increased risk of poorer general health. This association, however, may have been due to former drinkers who had quit because of ill health.

Looking at the frequency of alcohol drinking, men who drank fewer days per week had higher HRQOL levels for General Health, Vitality, and Mental Health. The Vitality score was also good for those who drank 3 to 4 and 5 to $6 \mathrm{~d} / \mathrm{wk}$. Although the association of the amount of alcohol consumption and its frequency with the HRQOL scales were slightly different, alcohol drinkers were more likely to rate their health as good in comparison with non-drinkers.

The HIPOP-OHP study demonstrated that alcohol drinking patterns were clearly associated with blood pressure levels ${ }^{24}$. Mean HDL-cholesterol levels were positively associated with alcohol consumption, which can have a protective effect on atherosclerosis ${ }^{2,3)}$. The reliability of the drinking assessment was moderate (kappa=0.76) for subjects who reported drinking status at two separate times in one year. Since it was possible that abstainers from alcohol drinking had a health problem, we analyzed them separately. But when several confounders, including ill health related to obesity, hypertension, hyperlipidemia, and diabetes were considered, the significant association of alcohol drinking with HRQOL was unchanged.

In the present study, we made the definition of sub- optimal HRQOL as less than the median score for each SF-36 sub-scale, similar to a previous study that considered "average," "rather poor," or "very poor" of five subjective health grades to be sub-optimal health ${ }^{17)}$. When SF-36 HRQOL scores were divided into quartiles and the lowest category was considered sub-optimal health, the associations of sub-optimal health with alcohol drinking were similarly demonstrated.

Our findings, based on the results of the physical and mental scales of the SF-36, were largely consistent with previous conclusions ${ }^{17,18)}$. That is, light and moderate alcohol drinking might have effects that modify the subjective experience of physical and mental health. Not only levels of alcohol consumption but also the frequency was similarly associated with the SF-36 scales, except for Role-Emotional. In the SF-36 validation study, scales of both Mental Health and Vitality were highly associated with mental conditions ${ }^{27}$. However, the Vitality condition remained at higher levels among men who drank more frequently.

The Japanese SF-36 validation study indicated that Role-Emotional represented physical condition rather than mental condition, as hypothesized, and its association with mental condition was dependent on the levels of psychiatric impairment ${ }^{27)}$. Given the difficulty in the interpretation of Role-Emotional, it is unclear why only Role-Emotional sub-scales were not associated with drinking status in our population.

Heavy drinkers were not at increased risk of suboptimal health in the present study, a finding contrary to the results from a general population study ${ }^{17}$. This may be explained by our population characteristics, in which occupational health was well managed. People with health problems were likely to quit or reduce drinking alcohol under intensive health management. Significant increased odds ratio of sub-optimal General Health for ex-drinkers supported in part this reasoning.

The strength of our study is the large population, which consists of mainly manufacturing and related company subjects/employees. This relatively homogeneous population helped us to interpret the effects of alcohol drinking on subjective health, including numerous factors related to working circumstances, carefully standardized in risk assessments. Nonetheless, several limitations should be considered. First, a cross-sectional study design does not prove causality. It can be argued that the data were biased by individuals who did not drink alcohol due to health problems, such as liver dysfunction. So, we separated abstainers from the analysis and presented the risks for sub-optimal health for the rest of the population. Furthermore, when we excluded subjects with levels of $\gamma$-GTP greater than $100 \mathrm{IU} / \mathrm{L}$ to rule out liver dysfunction, the associations remained. Second, the SF-36 NBS scores of our population were somewhat low in comparison with the national survey for SF-36 
standardization in 2002. Because subjective health is affected by socioeconomic status ${ }^{28)}$, we hypothesize that people with sub-optimal health may be over-represented due to the economic recession in Japan, especially for a workplace population such as in the present study. Third, reporting bias should be considered in the interpretations of the SF-36 sub-scales. For example, if people with favorable HRQOL levels are likely to underestimate their alcohol intake, our findings may have been to some extent affected by the bias. Nevertheless, it is impossible to rule it out from the present study design.

Although light and moderate alcohol consumption has often been reported to be most beneficial for cardiovascular disease and total mortality ${ }^{1-5)}$, alcohol drinking patterns also may provide benefits for subjective health, explained by the HRQOL sub-scales: RolePhysical, General Health, Vitality, and Mental Health. Nonetheless, a longitudinal study will be needed to clarify the potential causality of association between alcohol consumption and HRQOL conditions.

Acknowledgments: This study was funded by research grants from the Ministry of Health, Labour and Welfare, Japan (H10-12, No. 063, Research on Health Services, Health Sciences Research Grants, H13, No. 010 Medical Frontier Strategy Research, Health Sciences Research Grants) and from the Ministry of Health, Labour and Welfare, Japan (H14-16, No. 010 Clinical Research for Evidenced-Based Medicine, Health and Labour Sciences Research Grants). Also, this study was supported in part by the Japan Arteriosclerosis Prevention Fund 2000 and 2004 (Seikatsu 9).

\section{Appendix}

*HIPOP-OHP Research group:

Chairman: Hirotsugu Ueshima (Department of Health Science, Shiga University of Medical Science, Otsu, Shiga).

Participants: Akira Okayama (Department of Preventive Cardiology, National Cardiovascular Center, Osaka); Katsushi Yoshita (Department of National Nutrition Survey and Health Informatics, National Institute of Health and Nutrition); Toru Takebayashi and Yuriko Kikuchi (Department of Preventive Medicine and Public Health, School of Medicine, Keio University); Hideaki Nakagawa and Katsuyuki Miura (Department of Public Health, Kanazawa Medical University); Hiroshi Yamato (Institute of Industrial Ecological Science, University of Occupational and Environmental Health); Nagako Chiba (Department of Human-Life, Tsukuba International Junior College); Masahiko Yanagita (Faculty of Nursing and Social Welfare Sciences, Fukui Prefectural University); Kazunori Kodama, Fumiyoshi Kasagi and Nobuo Nishi; (Department of Epidemiology, Radiation Effects Research Foundation), Yukinori Kusaka
(Department of Environmental Health, Faculty of Medical Sciences, University of Fukui); Shigeyuki Saitoh (Second Department of Internal Medicine School of Medicine, Sapporo Medical University); Kiyomi Sakata (Department of Hygiene and Preventive Medicine, Iwate Medical University School of Medicine); Hideo Tanaka (Department of Cancer Control and Statistics, Osaka Medical Center for Cancer and Cardiovascular Diseases); Masakazu Nakamura (Cholesterol Reference Method Laboratory Network at Osaka Medical Center for Health Science and Promotion); Masakazu Nakamura (Osaka Medical Center for Health Science and Promotion); Yoshihiko Naito (Department of Food Sciences and Nutrition, Mukogawa Women's University); Yasuyuki Nakamura (Cardiovascular Epidemiology, Faculty of Home Economics, Kyoto Women's University); Makoto Watanabe and Yoshikazu Nakamura (Department of Public Health, Jichi Medical School); Akira Babazono (Institute of Health Science, Kyushu University), Unai Tamura, Junko Minai and Zentaro Yamagata (Department of Health Sciences, School of Medicine, University of Yamanashi); Sumio Urano (Matsushita Health Care Center), Fujihisa Kinoshita (Wakayama Wellness Foundation); Isao Saito (Department of Public Health, Nara Medical University); Shinichi Tanihara (Department of Public Health, Shimane University School of Medicine); Junko Tamaki (Department of Public Health, Kinki University School of Medicine); Osamu Tochikubo (Department of Public Health, Yokohama City University School of Medicine); Takeo Nakayama (Department of Medical System Informatics, Graduate School of Medicine and Faculty of Medicine, Kyoto University); Mariko Naito (Department of Preventive Medicine, Nagoya University Graduate School of Medicine); Shunichi Fukuhara and Yoshimi Suzukamo (Department of Epidemiology and Health Care Research, Graduate School of Medicine and Faculty of Medicine Kyoto University), Yoshiharu Fujieda (Department of Health and Sport Sciences, Tokyo Gakugei University); Shunsaku Mizushima (Department of Human Resources Development, National Institute of Public Health); Yuji Miyoshi (Tokyo Central Clinic, Health Insurance Society of Meiji Yasuda Life Insurance Company); Takayo Tada (Department of Food Science, Faculty of Human Life Science, Mimasaka University); Taichiro Tanaka, Takashi Kadowaki, Toshimi Yoshida, Mami Ide and Tomonori Okamura (Department of Health Science, Shiga University of Medical Science, Otsu, Shiga).

\section{References}

1) KJ Mukamal, KM Conigrave, MA Mittleman, CA Camargo Jr., MJ Stampfer, WC Willett and EB Rimm: Roles of drinking pattern and type of alcohol consumed in coronary heart disease in men. N Engl J Med 348, 109-118 (2003) 
2) H Iso, A Kitamura, T Shimamoto, T Sankai, Y Naito, S Sato, M Kiyama, M Iida and Y Komachi: Alcohol intake and the risk of cardiovascular disease in middleaged Japanese men. Stroke 26, 767-773 (1995)

3) A Kitamura, H Iso, T Sankai, Y Naito, S Sato, M Kiyama, T Okamura, Y Nakagawa, M Iida, T Shimamoto and Y Komachi: Alcohol intake and premature coronary heart disease in urban Japanese men. Am J Epidemiol 147, 59-65 (1998)

4) JM Gaziano, TA Gaziano, RJ Glynn, HD Sesso, UA Ajani, MJ Stampfer, JE Manson, $\mathrm{CH}$ Hennekens and JE Buring: Light-to-moderate alcohol consumption and mortality in the Physicians' Health Study enrollment cohort. J Am Coll Cardiol 35, 96-105 (2000)

5) S Tsugane, MT Fahey, S Sasaki and S Baba: Alcohol consumption and all-cause and cancer mortality among middle-aged Japanese men: seven-year follow-up of the JPHC study Cohort I. Japan Public Health Center. Am J Epidemiol 150, 1201-1207 (1999)

6) M Miyazaki and H Une: Japanese alcoholic beverage and all cause mortality in Japanese adult men. J Epidemiol 11, 219-223 (2001)

7) P Sillanaukee, T Koivula, H Jokela, H Myllyharju and K Seppa: Relationship of alcohol consumption to changes in HDL-subfractions. Eur J Clin Invest 23, 486-491 (1993)

8) SC Renaud, AD Beswick, AM Fehily, DS Sharp and PC Elwood: Alcohol and platelet aggregation: the caerphilly prospective heart disease study. Am J Clin Nutr 55, 1012-1017 (1992)

9) H Imano, H Iso, S Sato, A Kitamura, T Okamura, T Tanigawa, T Ohira, M Kudo, Y Naito, M Iida and T Shimamoto: Determinants of platelet aggregation in 50-70-year-old men from three Japanese communities. Atherosclerosis 165, 327-334 (2002)

10) LI Mennen, B Balkau, S Vol, E Caces and E Eschwege: Fibrinogen: a possible link between alcohol consumption and cardiovascular disease? DESIR Study Group. Arterioscler Thromb Vasc Biol 19, 887-892 (1999)

11) EL Idler, and Y Benyamini: Self-rated health and mortality: a review of twenty-seven community studies. J Health Soc Behav 38, 21-37 (1997)

12) S Heistaro, P Jousilahti, E Lahelma, E Vartiainen and P Puska: Self rated health and mortality: a long term prospective study in eastern Finland. J Epidemiol Community Health 55, 227-232 (2001)

13) NO Mansson and L Rastam: Self-rated health as a predictor of disability pension and death-a prospective study of middle-aged men. Scand J Public Health 29, 151-158 (2001)

14) J Heidrich, AD Liese, H Lowel and U Keil: Self-rated health and its relation to all-cause and cardiovascular mortality in southern Germany. Results from the MONICA Augsburg cohort study 1984-1995. Ann Epidemiol 12, 338-345 (2002)

15) NB Belloc and L Breslow: Relationship of physical health status and health practices. Prev Med 1, 409421 (1972)

16) L Breslow and JE Enstrom: Persistence of health habits and their relationship to mortality. Prev Med 9, 469483 (1980)

17) K Poikolainen, E Vartiainen and HJ Korhonen: Alcohol intake and subjective health. Am J Epidemiol 144, 346350 (1996)

18) C Power, B Rodgers and S Hope: U-shaped relation for alcohol consumption and health in early adulthood and implications for mortality. Lancet 352, 877 (1998)

19) M Gronbaek, EL Mortensen, K Mygind, AT Andersen, U Becker, C Gluud and TI Sorensen: Beer, wine, spirits and subjective health. J Epidemiol Community Health 53, 721-724 (1999)

20) Y Sakurai, N Hattori, T Kondo, K Teruya, N Shimada, S Honjo, T Umeda, T Muto, Y Takemura, I Todoroki and $\mathrm{K}$ Nakamura: Association between alcohol intake and subjective health: the Sotetsu Study. Keio J Med 48, 147-150 (1999)

21) MA Testa and DC Simonson: Assesment of qualityof-life outcomes. N Engl J Med 334, 835-840 (1996)

22) A Garratt, L Schmidt, A Mackintosh and R Fitzpatrick: Quality of life measurement: bibliographic study of patient assessed health outcome measures. BMJ 324, 1417 (2002)

23) T Okamura, T Tanaka, A Babazono, K Yoshita, N Chiba, T Takebayashi, H Nakagawa, H Yamato, K Miura, J Tamaki, T Kadowaki and A Okayama: The High-risk and Population Strategy for Occupational Health Promotion (HIPOP-OHP) study: study design and cardiovascular risk factors at the baseline survey. J Hum Hypertens 18, 475-485 (2004)

24) T Okamura, T Tanaka, K Yoshita, $\mathrm{N}$ Chiba, $\mathrm{T}$ Takebayashi, Y Kikuchi, J Tamaki, U Tamura, J Minai, T Kadowaki, K Miura, H Nakagawa, S Tanihara, A Okayama and H Ueshima: Specific alcoholic beverage and blood pressure in a middle-aged Japanese population: the High-risk and Population Strategy for Occupational Health Promotion (HIPOP-OHP) Study. J Hum Hypertens 18, 9-16 (2004)

25) Fukuhara S, Suzukamo T. Manual of SF-36v2 Japanese version. Kyoto: Institute for Health Outcomes \& Process Evaluation Research, 2004.

26) S Fukuhara, S Bito, J Green, A Hsiao and K Kurokawa: Translation, adaptation, and validation of the SF-36 Health Survey for use in Japan. J Clin Epidemiol 51, 1037-1044 (1998)

27) S Fukuhara, JE Ware Jr., M Kosinski, S Wada and B Gandek: Psychometric and clinical tests of validity of the Japanese SF-36 Health Survey. J Clin Epidemiol 51, 1045-1053 (1998)

28) H Hemingway, M Stafford, S Stansfeld, M Shipley and M Marmot: Is the SF-36 a valid measure of change in population health? Results from the Whitehall II Study. BMJ 315, 1273-1279 (1997) 